\title{
On the Correspondence between Classes of Implicational and Equivalence Quantifiers
}

\author{
Jiří Ivánek
}

Laboratory of Intelligent Systems, Faculty of Informatics and Statistics, University of Economics, W. Churchill Sq. 4, 13067 Prague, Czech Republic, e-mail: ivanek@vse.cz

\begin{abstract}
Relations between two Boolean attributes derived from data can be quantified by truth functions defined on four-fold tables corresponding to pairs of the attributes. In the paper, several classes of such quantifiers (implicational, double implicational, equivalence ones) with truth values in the unit interval are investigated. The method of construction of the logically nearest double implicational and equivalence quantifiers to a given implicational quantifier (and vice versa) is described and approved.
\end{abstract}

\section{Introduction}

The theory of observational quantifiers was established in the frame of the GUHA method of mechanized hypothesis formation [4, [5]. It should be stressed that this method is one of the earliest methods of data mining [9]. The method was during years developed and various procedures were implemented e.g. in the systems PC-GUHA [6], Knowledge Explorer [3], and 4FT-Miner [12. Further investigations of its mathematical and logical foundations are going on nowadays [7, [10, 11]. We concentrate to the most widely used observational quantifiers, called in [11 four-fold table quantifiers. So far this quantifiers were treated in classical logic as 0/1-truth functions. Some possibilities of fuzzy logic approach are now discussed [7].

In the paper, several classes of quantifiers (implicational, double implicational, equivalence ones) with truth values in the unit interval are investigated. Such type of quantifications of rules derived from databases is used in modern methods of knowledge discovery in databases (see e.g. [13]). On the other hand, there is a connection between four-fold table quantifiers and measures of resemblance or similarity applied on Boolean vectors [2.

In Section 2, basic notions and classes of quantifiers are defined, and some examples of quantifiers of different types are given. In Section 3, the method of construction of double implicational quantifiers from implicational ones (and vice versa) is described. This method provides a logically strong one-to-one correspondence between classes of implicational and so called $\Sigma$-double implicational quantifiers. An analogical construction is used in Section 4 to introduce similar correspondence between classes of $\Sigma$-double implicational and $\Sigma$-equivalence quantifiers. Several theorems on this constructions are proved. As a conclusion, triads of affiliated quantifiers are introduced, and their importance in data mining applications is discussed. 


\section{Classes of Quantifiers}

For two Boolean attributes $\varphi$ and $\psi$ (derived from given data), corresponding four-fold table $\langle a, b, c, d\rangle$ (Table 1 ) is composed from numbers of objects in data satisfying four different Boolean combinations of attributes:

$a$ is the number of objects satisfying both $\varphi$ and $\psi$,

$b$ is the number of objects satisfying $\varphi$ and not satisfying $\psi$,

$c$ is the number of objects not satisfying $\varphi$ and satisfying $\psi$,

$d$ is the number of objects not satisfying $\varphi$ and not satisfying $\psi$.

\begin{tabular}{r|c|c} 
& $\psi$ & $\neg \psi$ \\
\hline$\varphi$ & $a$ & $b$ \\
\hline$\neg \varphi$ & $c$ & $d$
\end{tabular}

Table 1. Four-fold table of $\varphi$ and $\psi$

To avoid degenerated situations, we shall assume, that all marginals of the four-fold table are non-zero:

$a+b>0, c+d>0, a+c>0, b+d>0$.

Definition 1. 4FT quantifier $\sim$ is a $[0,1]$-valued function defined for all fourfold tables $\langle a, b, c, d\rangle$. We shall write $\sim(a, b)$ if the value of the quantifier $\sim$ depends only on $a, b ; \sim(a, b, c)$ if the value of the quantifier $\sim$ depends only on $a, b, c ; \sim(a, b, c, d)$ if the value of the quantifier $\sim$ depends on all $a, b, c, d$. For simplicity, we shall omit in this paper specification $4 F T$.

The most common examples of quantifiers are following ones:

Example 1. Quantifier $\Rightarrow \oslash$ of basic implication (corresponds to the notion of a confidence of an association rule, see [1], 4], [5]):

$\Rightarrow_{\oslash}(a, b)=\frac{a}{a+b}$.

Example 2. Quantifier $\Leftrightarrow \oslash$ of basic double implication (Jaccard 1900, [2], [5]):

$\Leftrightarrow \oslash(a, b, c)=\frac{a}{a+b+c}$.

Example 3. Quantifier $\equiv_{\oslash}$ of basic equivalence (Kendall, Sokal-Michener 1958, [2], [5]):

$$
\equiv(a, b, c, d)=\frac{a+d}{a+b+c+d} \text {. }
$$

If the four-fold table $\langle a, b, c, d\rangle$ represents the behaviour of the derived attributes $\varphi$ and $\psi$ in given data, then we can interpret above quantifiers in the following way:

The quantifier of basic implication calculates the relative frequency of objects satisfying $\psi$ out from all objects satisfying $\varphi$, so it is measuring in a simple way 
the validity of implication $\varphi \Rightarrow \psi$ in data. The higher is $a$ and the smaller is $b$, the better is validity $\Rightarrow_{\oslash}(a, b)=\frac{a}{a+b}$.

The quantifier of basic double implication calculates the relative frequency of objects satisfying $\varphi \wedge \psi$ out from all objects satisfying $\varphi \vee \psi$, so it is measuring in a simple way the validity of bi-implication $(\varphi \Rightarrow \psi) \wedge(\psi \Rightarrow \varphi)$ in data. The higher is $a$ and the smaller are $b, c$, the better is validity $\Leftrightarrow_{\oslash}(a, b, c)=\frac{a}{a+b+c}$.

The quantifier of basic equivalence calculates the relative frequency of objects supporting correlation of $\varphi$ and $\psi$ out from all objects, so it is measuring in a simple way the validity of equivalency $\varphi \equiv \psi$ in data. The higher is $a, d$ and the smaller are $b, c$, the better is validity $\equiv \oslash(a, b, c, d)=\frac{a+d}{a+b+c+d}$.

Properties of basic quantifiers are in the core of the general definition of several useful classes of quantifiers [4, 5], [11,

(1) $I$ - class of implicational quantiffiers,

(2) $D I$ - class of double implicational quantiffiers,

(3) $\Sigma D I$ - class of $\Sigma$-double implicational quantiffiers,

(4) $E$ - class of equivalence quantiffiers,

(5) $\Sigma E$ - class of $\Sigma$-equivalence quantiffiers.

Each class of quantifiers $\sim$ is characterized in the following definition by a special truth preservation condition of the form: fact that the four-fold table $\left.<a^{\prime}, b^{\prime}, c^{\prime}, d^{\prime}\right\rangle$ is in some sense (implicational, ...) better than $\langle a, b, c, d\rangle$ implies that $\sim\left(a^{\prime}, b^{\prime}, c^{\prime}, d^{\prime}\right) \geq \sim(a, b, c, d)$.

Definition 2. Let $a, b, c, d, a^{\prime}, b^{\prime}, c^{\prime}, d^{\prime}$ mean frequencies from arbitrary pairs of four-fold tables $\langle a, b, c, d\rangle$ and $\left\langle a^{\prime}, b^{\prime}, c^{\prime}, d^{\prime}\right\rangle$.

(1) A quantifier $\sim(a, b)$ is implicational, $\sim \in I$, if always

$a^{\prime} \geq a \wedge b^{\prime} \leq b \quad$ implies $\sim\left(a^{\prime}, b^{\prime}\right) \geq \sim(a, b)$.

(2) A quantifier $\sim(a, b, c)$ is double implicational, $\sim \in D I$, if always

$a^{\prime} \geq a \wedge b^{\prime} \leq b \wedge c^{\prime} \leq c$ implies $\sim\left(a^{\prime}, b^{\prime}, c^{\prime}\right) \geq \sim(a, b, c)$.

(3) A quantifier $\sim(a, b, c)$ is $\Sigma$-double implicational, $\sim \in \Sigma D I$, if always $a^{\prime} \geq a \wedge b^{\prime}+c^{\prime} \leq b+c$ implies $\sim\left(a^{\prime}, b^{\prime}, c^{\prime}\right) \geq \sim(a, b, c)$.

(4) A quantifier $\sim(a, b, c, d)$ is equivalence, $\sim \in E$, if always $a^{\prime} \geq a \wedge b^{\prime} \leq b \wedge c^{\prime} \leq c \wedge d^{\prime} \geq d$ implies $\sim\left(a^{\prime}, b^{\prime}, c^{\prime}, d^{\prime}\right) \geq \sim(a, b, c, d)$.

(5) A quantifier $\sim(a, b, c, d)$ is $\Sigma$-equivalence, $\sim \in \Sigma E$, if always $a^{\prime}+d^{\prime} \geq a+d \wedge b^{\prime}+c^{\prime} \leq b+c$ implies $\sim\left(a^{\prime}, b^{\prime}, c^{\prime}, d^{\prime}\right) \geq \sim(a, b, c, d)$.

Example 4. $\Rightarrow_{\oslash} \in I, \Leftrightarrow_{\oslash} \in \Sigma D I, \equiv_{\oslash} \in \Sigma E$.

\section{Proposition 3. $I \subset D I \subset E, \Sigma D I \subset D I, \Sigma E \subset E$.}

In the original GUHA method [4, [5], some statistically motivated quantifiers were introduced. They are based on hypotheses testing, e.g.: Given $0<p<1$, the question is if the conditional probability corresponding to the examined relation of Boolean attributes $\varphi$ and $\psi$ is $\geq p$. This question lead to the test of the null hypothesis that corresponding conditional probability is $\geq p$, against the alternative hypothesis that this probability is $<p$. The following quantifiers are derived from the appropriate statistical test. 
Example 5. Quantifier $\Rightarrow_{p}^{?}$ of upper critical implication

$$
\Rightarrow_{p}^{?}(a, b)=\sum_{i=0}^{a} \frac{(a+b) !}{i !(a+b-i) !} p^{i}(1-p)^{a+b-i}
$$

is implicational [4], [5].

Example 6. Quantifier $\Leftrightarrow_{p}^{?}$ of upper critical double implication

$$
\Leftrightarrow \stackrel{?}{p}(a, b, c)=\sum_{i=0}^{a} \frac{(a+b+c) !}{i !(a+b+c-i) !} p^{i}(1-p)^{a+b+c-i}
$$

is $\Sigma$-double implicational [5],[11].

Example \%. Quantifier $\equiv_{p}^{?}$ of upper critical equivalence

$$
\equiv_{p}^{?}(a, b, c, d)=\sum_{i=0}^{a+d} \frac{(a+b+c+d) !}{i !(a+b+c+d-i) !} p^{i}(1-p)^{a+b+c+d-i}
$$

is $\Sigma$-equivalence [5], 11].

Let us note, that all the above mentioned quantifiers are used (among others) in the GUHA procedure 4FT-Miner [12].

Some more examples of double implicational and equivalence quantifiers can be derived from the list of association coefficients (resemblance measures on Boolean vectors) included in [2].

In the next sections, one-to-one correspondence with strong logical properties will be shown

i) between classes of quantifiers $I, \Sigma D I$ by means of the relation: $\Leftrightarrow^{*}(a, b, c)=\Rightarrow^{*}(a, b+c)$,

and, analogously,

ii) between classes of quantifiers $\Sigma D I, \Sigma E$ by means of the relation: $\equiv^{*}(a, b, c, d)=\Leftrightarrow^{*}(a+d, b, c)$.

First, let us prove the following auxiliary propositions.

Lemma 4. A quantifier $\Leftrightarrow^{*}$ is $\Sigma$-double implicational iff the following conditions hold:

(i) for all $a, b, c, b^{\prime}, c^{\prime}$ such that $b^{\prime}+c^{\prime}=b+c$

$\Leftrightarrow^{*}\left(a, b^{\prime}, c^{\prime}\right)=\Leftrightarrow^{*}(a, b, c)$ holds,

(ii) the quantifier $\Rightarrow^{*}$ defined by $\Rightarrow^{*}(a, b)=\Leftrightarrow^{*}(a, b, 0)$ is implicational.

Proof. For $\Sigma$-double implicational quantifiers, (i), (ii) are clearly true. Let $\Leftrightarrow^{*}$ is a quantifier satisfying (i), (ii), and $a^{\prime} \geq a \wedge b^{\prime}+c^{\prime} \leq b+c$. Then

$\Leftrightarrow^{*}\left(a^{\prime}, b^{\prime}, c^{\prime}\right)=\Leftrightarrow^{*}\left(a^{\prime}, b^{\prime}+c^{\prime}, 0\right)=\Rightarrow^{*}\left(a^{\prime}, b^{\prime}+c^{\prime}\right) \geq$

$\geq \Rightarrow^{*}(a, b+c)=\Leftrightarrow^{*}(a, b+c, 0)=\Leftrightarrow^{*}(a, b, c)$. 
Example 8. Quantifier $\Leftrightarrow_{+}$(Kulczynski 1927, see [2]):

$\Leftrightarrow_{+}(a, b, c)=\frac{1}{2}\left(\frac{a}{a+b}+\frac{a}{a+c}\right)$

is double implicational but not $\Sigma$-double implicational, $\Leftrightarrow_{+} \in D I-\Sigma D I$; for instance $\Leftrightarrow_{+}(1,1,1)=\Leftrightarrow_{+}(1,2,0)$ does not hold.

Lemma 5. A quantifier $\equiv^{*}$ is $\Sigma$-equivalence iff the following conditions hold:

(i) for all $a, b, c, d, a^{\prime}, b^{\prime}, c^{\prime}, d^{\prime}$ such that $a^{\prime}+d^{\prime}=a+d, b^{\prime}+c^{\prime}=b+c$ $\equiv^{*}\left(a^{\prime}, b^{\prime}, c^{\prime}, d^{\prime}\right)=\equiv^{*}(a, b, c, d)$ holds,

(ii) the quantifier $\Leftrightarrow^{*}$ defined by $\Leftrightarrow^{*}(a, b, c)=\equiv^{*}(a, b, c, 0)$ is $\Sigma$-double implicational.

Proof. For $\Sigma$-equivalence quantifiers, (i), (ii) are clearly true. Let $\equiv^{*}$ is a quantifier satisfying (i), (ii), and $a^{\prime}+d^{\prime} \geq a+d \wedge b^{\prime}+c^{\prime} \leq b+c$. Then

$$
\begin{aligned}
& \equiv^{*}\left(a^{\prime}, b^{\prime}, c^{\prime}, d^{\prime}\right)=\equiv^{*}\left(a^{\prime}+d^{\prime}, b^{\prime}, c^{\prime}, 0\right)=\Leftrightarrow^{*}\left(a^{\prime}+d^{\prime}, b^{\prime}, c^{\prime}\right) \geq \\
& \geq \Leftrightarrow^{*}(a+d, b, c)=\equiv^{*}(a+d, b, c)=\equiv^{*}(a, b, c, d) .
\end{aligned}
$$

Example 9. Quantifier $\equiv_{+}$(Sokal, Sneath 1963, see [2]):

$$
\equiv_{+}(a, b, c, d)=\frac{1}{4}\left(\frac{a}{a+b}+\frac{a}{a+c}+\frac{d}{d+b}+\frac{d}{d+c}\right)
$$

is equivalence but not $\Sigma$-equivalence, $\equiv_{+} \in E-\Sigma E$;

for instance $\equiv_{+}(1,1,1,1)=\equiv_{+}(2,1,1,0)$ does not hold.

We shall use the following definition to state relations between different quantifiers:

Definition 6. A quantifier $\sim_{1}$ is less strict than $\sim_{2}$ (or $\sim_{2}$ is more strict than $\sim_{1}$ ) if for all four-fold tables $\langle a, b, c, d\rangle$

$\sim_{1}(a, b, c, d) \geq \sim_{2}(a, b, c, d)$.

From the (fuzzy) logic point of view, it means that in all models (data) the formula $\varphi \sim_{1} \psi$ is at least so true as the formula $\varphi \sim_{2} \psi$, i.e. the deduction rule $\frac{\varphi \sim_{2} \psi}{\varphi \sim_{1} \psi}$ is correct.

Example 10. $\Leftrightarrow_{\oslash}$ is more strict than $\Rightarrow_{\varnothing}$, and less strict than $\equiv_{\varnothing}$;

$\Leftrightarrow_{+}$is more strict than $\Leftrightarrow_{\oslash}$.

\section{Correspondence between Classes of $\boldsymbol{\Sigma}$-Double Implicational Quantifiers and Implicational Ones}

Let $\Rightarrow^{*}$ be an implicational quantifier. There is a natural task to construct some $\Sigma$-double implicational quantifier $\Leftrightarrow^{*}$ such that from formula $\varphi \Leftrightarrow^{*} \psi$ logically follow both implications $\varphi \Rightarrow^{*} \psi, \psi \Rightarrow^{*} \varphi$, i.e. deduction rules $\frac{\varphi \Leftrightarrow^{*} \psi}{\varphi \Rightarrow^{*} \psi}, \frac{\varphi \Leftrightarrow *}{\psi \Rightarrow^{*} \varphi}$ are correct. Such a quantifier $\Leftrightarrow^{*}$ should be as less strict as possible to be near to $\Rightarrow^{*}$.

Following two theorems show how to construct the logically nearest $\Sigma$-double implicational quantifier from a given implicational quantifier and vice versa. 
Theorem 7. Let $\Rightarrow^{*}$ be an implicational quantifier and $\Leftrightarrow^{*}$ be the quantifier constructed from $\Rightarrow^{*}$ for all four-fold tables $\langle a, b, c, d\rangle$ by the formula $\Leftrightarrow^{*}(a, b, c)=\Rightarrow^{*}(a, b+c)$.

Then $\Leftrightarrow^{*}$ is the $\Sigma$-double implicational quantifier which is the least strict from the class of all $\Sigma$-double implicational quantifiers $\sim$ satisfying for all four-fold tables $\langle a, b, c, d\rangle$ the property

$\sim(a, b, c) \leq \min \left(\Rightarrow^{*}(a, b), \Rightarrow^{*}(a, c)\right)$.

Remark. Let us mention that this means the following:

(1) deduction rules $\frac{\varphi \Leftrightarrow{ }^{*} \psi}{\varphi \Rightarrow^{*} \psi}, \frac{\varphi \Leftrightarrow{ }^{*} \psi}{\psi \Rightarrow^{*} \varphi}$ are correct;

(2) if $\sim$ is a $\Sigma$-double implicational quantifier such that deduction rules

$\frac{\varphi \sim \psi}{\varphi \Rightarrow^{*} \psi}, \frac{\varphi \sim \psi}{\psi \Rightarrow^{*} \varphi}$ are correct,

then $\sim$ is more strict than $\Leftrightarrow^{*}$, i.e. also $\frac{\varphi \sim \psi}{\varphi \Leftrightarrow^{*} \psi}$ is correct.

Proof. Since $\Rightarrow^{*}$ is an implicational quantifier, $\Leftrightarrow^{*}$ is a $\Sigma$-double implicational quantifier; moreover,

$\Leftrightarrow^{*}(a, b, c)=\Rightarrow^{*}(a, b+c) \leq \min \left(\Rightarrow^{*}(a, b), \Rightarrow^{*}(a, c)\right)$

for all four-fold tables $\langle a, b, c, d\rangle$.

Let $\sim$ is a $\Sigma$-double implicational quantifier satisfying the property

$\sim(a, x, y) \leq \min \left(\Rightarrow^{*}(a, x), \Rightarrow^{*}(a, y)\right)$

for all four-fold tables $\langle a, x, y, d\rangle$.

Then we obtain using Lemma 4

$\sim(a, b, c)=\sim(a, b+c, 0) \leq \Rightarrow^{*}(a, b+c)=\Leftrightarrow^{*}(a, b, c)$

for all four-fold tables $\langle a, b, c, d\rangle$, which means that $\sim$ is more strict than $\Leftrightarrow^{*}$.

Example 11. (1) For the basic implication $\Rightarrow_{\oslash}(a, b)=\frac{a}{a+b}$, the basic double implication $\Leftrightarrow_{\oslash}(a, b, c)=\frac{a}{a+b+c}$ is the least strict $\Sigma$-double implicational quantifier satisfying deduction rules $\frac{\varphi \Leftrightarrow * \psi}{\varphi \Rightarrow \oslash \psi}, \frac{\varphi \Leftrightarrow^{*} \psi}{\psi \Rightarrow \oslash \varphi}$.

(2) For the upper critical implication

$\Rightarrow \stackrel{?}{p}(a, b)=\sum_{i=0}^{a} \frac{(a+b) !}{i !(a+b-i) !} p^{i}(1-p)^{a+b-i}$,

the upper critical double implication

$\Leftrightarrow{ }_{p}^{?}(a, b, c)=\sum_{i=0}^{a} \frac{(a+b+c) !}{i !(a+b+c-i) !} p^{i}(1-p)^{a+b+c-i}$

is the least strict $\Sigma$-double implicational quantifier satisfying deduction rules $\frac{\varphi \Leftrightarrow{ }^{*} \psi}{\varphi \Rightarrow{ }_{p}^{?} \psi}, \frac{\varphi \Leftrightarrow{ }^{*} \psi}{\psi \Rightarrow{ }_{p}^{?} \varphi}$.

Theorem 8. Let $\Leftrightarrow^{*}$ be a $\Sigma$-double implicational quantifier and $\Rightarrow^{*}$ be the quantifier constructed from $\Leftrightarrow^{*}$ for all four-fold tables $\langle a, b, c, d\rangle$ by the formula

$\Rightarrow^{*}(a, b)=\Leftrightarrow^{*}(a, b, 0)$.

Then $\Rightarrow^{*}$ is the implicational quantifier which is the most strict from the class of all implicational quantifiers $\sim$ satisfying for all four-fold tables $\langle a, b, c, d\rangle$ the property

$$
\min (\sim(a, b), \sim(a, c)) \geq \Leftrightarrow^{*}(a, b, c) .
$$


Remark. Let us mention that this means the following:

(1) deduction rules $\frac{\varphi \Leftrightarrow^{*} \psi}{\varphi \Rightarrow^{*} \psi}, \frac{\varphi \Leftrightarrow^{*} \psi}{\psi \Rightarrow^{*} \varphi}$ are correct;

(2) if $\sim$ is an implicational quantifier such that deduction rules $\frac{\varphi \Leftrightarrow * \psi}{\varphi \sim \psi}, \frac{\varphi \Leftrightarrow^{*} \psi}{\psi \sim \varphi}$ are correct,

then $\sim$ is less strict than $\Rightarrow^{*}$, i.e. also $\frac{\varphi \Rightarrow^{*} \psi}{\varphi \sim \psi}$ is correct.

Proof. Since $\Leftrightarrow^{*}$ is a $\Sigma$-double implicational quantifier, $\Rightarrow^{*}$ is an implicational quantifier; moreover,

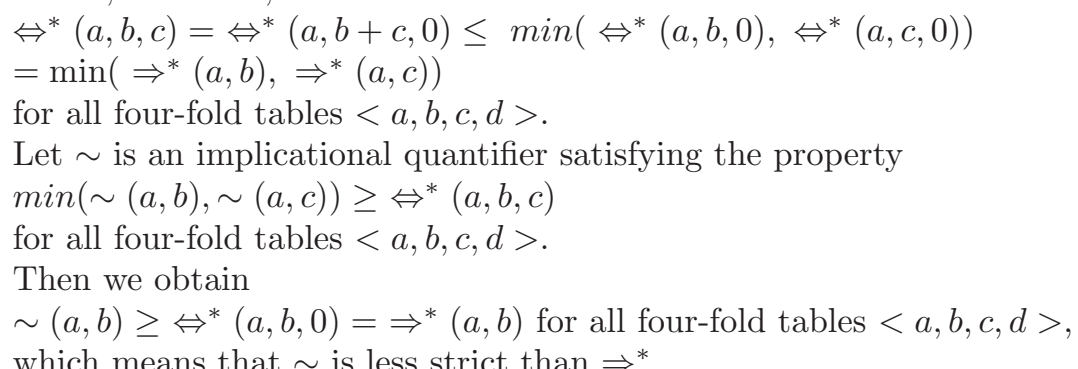

\section{Correspondence between Classes of $\Sigma$-Equivalence Quantifiers and $\Sigma$-Double Implicational Ones}

This section will be a clear analogy with the previous one:

Let $\Leftrightarrow^{*}$ be an $\Sigma$-double implicational quantifier. There is a natural task to construct some $\Sigma$-equivalence ${ }^{*}$ such that the formula $\varphi \equiv{ }^{*} \psi$ logically follows both from the formula $\varphi \Leftrightarrow^{*} \psi$, and from the formula $\neg \varphi \Leftrightarrow^{*} \neg \psi$, i.e. deduction rules $\frac{\varphi \Leftrightarrow^{*} \psi}{\varphi \equiv{ }^{*} \psi}, \frac{\neg \varphi \Leftrightarrow^{*} \neg \psi}{\varphi \equiv^{*} \psi}$ are correct. Such a quantifier $\equiv^{*}$ should be as strict as possible to be near to $\Leftrightarrow^{*}$.

Following theorems show how to construct the logically nearest $\Sigma$-equivalence quantifier from a given $\Sigma$-double implicational quantifier and vice versa. The proofs of these theorems are similar to the proofs of Theorems 7,8, so we shall omit them for the lack of space.

Theorem 9. Let $\Leftrightarrow^{*}$ be a $\Sigma$-double implicational quantifier and $\equiv^{*}$ be the quantifier constructed from $\Leftrightarrow^{*}$ for all four-fold tables $\langle a, b, c, d\rangle$ by the formula $\equiv^{*}(a, b, c, d)=\Leftrightarrow^{*}(a+d, b, c)$.

Then ${ }^{*}$ is the $\Sigma$-equivalence which is the most strict from the class of all $\Sigma$-equivalences $\sim$ satisfying for all four-fold tables $\langle a, b, c, d\rangle$ the property $\sim(a, b, c, d) \geq \max \left(\Leftrightarrow^{*}(a, b, c), \Leftrightarrow^{*}(d, b, c)\right)$.

Example 12. (1) For the basic double implication $\Leftrightarrow_{\oslash}(a, b, c)=\frac{a}{a+b+c}$, the basic equivalence $\equiv \oslash(a, b, c, d)=\frac{a+d}{a+b+c+d}$, is the most strict $\Sigma$-equivalence satisfying deduction rules $\frac{\varphi \Leftrightarrow \oslash \psi}{\varphi \equiv{ }^{*} \psi}, \frac{\neg \varphi \Leftrightarrow \oslash \neg \psi}{\varphi \equiv{ }^{*} \psi}$.

(2) For the upper critical double implication $\Leftrightarrow \stackrel{?}{p}^{\prime}(a, b, c)=\sum_{i=0}^{a} \frac{(a+b+c) !}{i !(a+b+c-i) !} p^{i}(1-p)^{a+b+c-i}$, 
the upper critical equivalence

$\equiv_{p}^{?}(a, b, c, d)=\sum_{i=0}^{a+d} \frac{(a+b+c+d) !}{i !(a+b+c+d-i) !} p^{i}(1-p)^{a+b+c+d-i}$

is the most strict $\Sigma$-equivalence satisfying deduction rules $\frac{\varphi \Leftrightarrow{ }_{p}^{?} \psi}{\varphi \equiv^{*} \psi}, \frac{\neg \varphi \Leftrightarrow ?{ }_{p}^{?} \neg \psi}{\varphi \equiv^{*} \psi}$.

Theorem 10. Let $\equiv^{*}$ be an $\Sigma$-equivalence quantifier and $\Leftrightarrow^{*}$ be the quantifier constructed from $\equiv^{*}$ for all four-fold tables $\langle a, b, c, d\rangle$ by the formula $\Leftrightarrow^{*}(a, b, c)=\equiv^{*}(a, b, c, 0)$.

Then $\Leftrightarrow^{*}$ is the $\Sigma$-double implicational quantifier which is the least strict from the class of all $\Sigma$-double implicational quantifiers $\sim$ satisfying for all four-fold tables $\langle a, b, c, d\rangle$ the property

$\max (\sim(a, b, c), \sim(d, b, c)) \leq \equiv^{*}(a, b, c, d)$.

\section{Conclusions}

The theorems proved in the paper show that quantifiers from classes $I, \Sigma D I, \Sigma E$ compose logically affiliated triads $\Rightarrow^{*}, \Leftrightarrow^{*}, \equiv^{*}$, where

$\Rightarrow^{*}$ is implicational quantifier,

$\Leftrightarrow^{*}$ is $\Sigma$-double implicational quantifier,

$\equiv^{*}$ is $\Sigma$-equivalence.

Examples of such triads included in this paper are:

Example 13. Triad of basic quantifiers $\Rightarrow_{\oslash}, \Leftrightarrow_{\oslash}, \equiv_{\oslash}$, where

$\Rightarrow \oslash(a, b)=\frac{a}{a+b}, \quad \Leftrightarrow \oslash(a, b, c)=\frac{a}{a+b+c}, \quad \equiv \oslash(a, b, c, d)=\frac{a+d}{a+b+c+d}$.

Example 14. Triad of statistically motivated upper critical quantifiers

$\Rightarrow ? \stackrel{?}{p}, \Leftrightarrow{ }_{p}^{?}, \equiv_{p}^{?}$, where

$\Rightarrow_{p}^{?}(a, b)=\sum_{i=0}^{a} \frac{(a+b) !}{i !(a+b-i) !} p^{i}(1-p)^{a+b-i}$,

$\Leftrightarrow{ }_{p}^{?}(a, b, c)=\sum_{i=0}^{a} \frac{(a+b+c) !}{i !(a+b+c-i) !} p^{i}(1-p)^{a+b+c-i}$,

$\equiv_{p}^{?}(a, b, c, d)=\sum_{i=0}^{a+d} \frac{(a+b+c+d) !}{i !(a+b+c+d-i) !} p^{i}(1-p)^{a+b+c+d-i}$.

Let us stress that to each given quantifier from classes $I, \Sigma D I, \Sigma E$, such triad can be constructed. This can naturally extend the metodological approach used to the particular quantifier's definition for covering all three types of relations (implication, double implication, equivalence).

We proved that following deduction rules are correct for the triads:

$$
\frac{\varphi \Leftrightarrow^{*} \psi}{\varphi \Rightarrow^{*} \psi}, \frac{\varphi \Leftrightarrow^{*} \psi}{\psi \Rightarrow^{*} \varphi}, \frac{\varphi \Leftrightarrow^{*} \psi}{\varphi \equiv^{*} \psi}, \frac{\neg \varphi \Leftrightarrow^{*} \neg \psi}{\varphi \equiv^{*} \psi} .
$$

These deduction rules can be used in knowledge discovery and data mining methods in various ways:

(1) to organize efectively search for rules in databases (discovering some rules is a reason to skip over in search, because of some other rules simply follows from 
discovered ones; nonvalidity of some rules means that some others are also non valid, ...);

(2) to filter results of data mining procedure (results which follows from others are not so interesting for users);

(3) to order rules according different (but affiliated) quantifications.

In practice, some of the above described ideas were used in the systems Combinational Data Analysis, ESOD [8], Knowledge Explorer 3], and 4FTMiner [12].

This research has been supported by grant VS96008 of the Ministry of Education, Youth and Sports of the Czech Republic. The author is grateful to J.Rauch and R.Jiroušek for their valuable comments on the preliminary version of the paper.

\section{References}

1. Aggraval, R. et al.: Fast Discovery of Association Rules. In Fayyad, V.M. et al.: Advances in Knowledge Discovery and Data Mining. AAAI Press / MIT Press 1996, p.307-328.

2. Batagelj, V., Bren, M.: Comparing Resemblance Measures. J. of Classification 12 (1995), p. 73-90.

3. Berka, P., Ivánek, J.: Automated Knowledge Acquisition for PROSPECTOR-like Expert Systems. In Machine Learning. ECML-94 Catania (ed. Bergadano, Raedt). Springer 1994, p.339-342.

4. Hájek,P., Havránek,T.: Mechanising Hypothesis Formation - Mathematical Foundations for a General Theory. Springer-Verlag, Berlin 1978, 396 p.

5. Hájek,P., Havránek,T., Chytil M.: Metoda GUHA. Academia, Praha 1983, 314 p. (in Czech)

6. Hájek, P., Sochorová, A., Zvárová, J.: GUHA for personal computers. Computational Statistics \& Data Analysis 19 (1995), p. 149 - 153

7. Hájek, P., Holeňa, M.: Formal Logics of Discovery and Hypothesis Formation by Machine. In Discovery Science (Arikawa,S. and Motoda,H., eds.), Springer-Verlag, Berlin 1998, p.291-302

8. Ivánek, J., Stejskal, B.: Automatic Acquisition of Knowledge Base from Data without Expert: ESOD (Expert System from Observational Data). In Proc. COMPSTAT'88 Copenhagen. Physica-Verlag, Heidelberg 1988, p.175-180.

9. Rauch,J.: GUHA as a Data Mining Tool. In: Practical Aspects of Knowledge Management. Schweizer Informatiker Gesellshaft Basel, 1996

10. Rauch, J.: Logical Calculi for Knowledge Discovery in Databases. In Principles of Data Mining and Knowledge Discovery, (Komorowski,J. and Zytkow,J., eds.), Springer-Verlag, Berlin 1997, p. 47-57.

11. Rauch,J.: Classes of Four-Fold Table Quantifiers. In Principles of Data Mining and Knowledge Discovery, (Quafafou,M. and Zytkow,J., eds.), Springer Verlag, Berlin 1998, p. 203-211.

12. Rauch,J.: 4FT-Miner - popis procedury. Technical Report LISp-98-09, Praha 1999.

13. Zembowicz,R. - Zytkow,J.: From Contingency Tables to Various Forms of Knowledge in Databases. In Fayyad, U.M. et al.: Advances in Knowledge Discovery and Data Mining. AAAI Press/ The MIT Press 1996, p. 329-349. 